\title{
Stanley John Dudrick titán de la cirugía y la nutrición parenteral: definiendo la tecnología disruptiva
}

\author{
Stanley John Dudrick titan of surgery and parenteral nutrition: \\ defining disruptive technology \\ Stanley John Dudrick, titã em cirurgia e nutrição parenteral: \\ definindo a tecnologia disruptiva
}

José Mario Pimiento*

https://doi.org/10.35454/rncm.v3n1.036

Imaginemos un paciente de 50 años con eritema en la herida quirúrgica, en el quinto día postoperatorio después de una laparotomía exploratoria durante la cual el paciente requirió múltiples resecciones intestinales como resultado de una hernia ventral encancerada crónica y sintomática por varias semanas. Imaginemos que el paciente estaba recibiendo dieta líquida y esperando el regreso de la función intestinal. Imaginemos que la albúmina sérica es $2,5 \mathrm{mg} / \mathrm{dL}$ y que el paciente presenta evidencia de anasarca. Imaginemos también que al explorar la herida quirúrgica se encuentra contenido intestinal y se diagnostica una fístula entero-atmosférica. En la medicina moderna esta situación clínica que presenta el paciente es un reto, pero no una sentencia a muerte. Más aún, en la actualidad los hospitales de alta complejidad cuentan con grupos multidisciplinarios especializados en el tratamiento de pacientes con este tipo de complicaciones.

Recordemos que cuando no existía la nutrición parenteral, los pacientes que no podían alimentarse por vía enteral estaban condenados a morir de inanición. A propósito, permítanme contarles una historia: en los años sesenta un residente de primer año de cirugía, un interno, en el hospital de Pensilvania estuvo pensando en cambiarse de especialidad porque pacientes con cuadros

* Jose.Pimiento@moffitt.org clínicos como el mencionado arriba no se recuperaban de estas cirugías "exitosas" y morían en el postoperatorio $^{(1)}$. Este residente era Stanley J. Dudrick, un joven de origen humilde, nacido en la región de las minas de carbón al oeste de Pensilvania. Este cirujano en entrenamiento trabajó de manera incansable, y en contra de la sabiduría popular, para desarrollar un método de nutrir a los pacientes por vía intravenosa. Su mentor el doctor Jonathan E. Rhoads, le dio acceso a su laboratorio en donde pudo colaborar con el doctor Harry M. Vars, una autoridad en farmacología ${ }^{(1,2)}$, y de esta manera alcanzar su potencial y cambiar el mundo.

Es importante recordar que en el ambiente académico de mediados del siglo pasado la nutrición intravenosa total se consideraba un problema insuperable con una solución inalcanzable, un auténtico "nudo Gordiano": "Primero se decía, que era un problema imposible de resolver, si fuera posible resolverlo no se podría implementar, y si se pudiera implementar sería demasiado costoso hacerlo" ${ }^{(2)}$. El trabajo fue tedioso y nada glamuroso, pero la dedicación, la creatividad, la adaptabilidad y la obstinación del doctor Dudrick lo llevaron a solucionar cada uno de los problemas que no habían sido resueltos hasta el momento y que hicieron posible el desarrollo de una nueva tecnología: la Nutrición Parenteral Total.

La mezcla de nutrientes, a partir de soluciones que tuvieran concentraciones adecuadas de dextrosa hipertónica y de hidrosilato de proteína ( 1.000 calorías y 6 
g proteína por litro, o $1 \mathrm{cal} / \mathrm{mL})^{(2)}$, que además fuera químicamente estable, fue un proceso largo y lleno de obstáculos que pudieron hacer que el doctor Dudrick renunciara a su proyecto. Sin embargo, una vez conquistó este reto inicial, imposible, empezaron los problemas reales. Fue la forma como el doctor Dudrick resolvió estos problemas lo que marcó la diferencia entre el genio y las demás personas que intentaron la Nutrición Parenteral Total antes que él.

Tecnología disruptiva es definida como aquella innovación que altera la manera en la que una industria actúa ${ }^{(3)}$, en este caso la industria de la medicina. Lograr que la Nutrición Parenteral Total fuera una realidad, requirió una serie de innovaciones y avances tecnológicos, que han llevado a considerarla y a definirla como Tecnología Disruptiva en Medicina como pocos avances en la historia lo han podido hacer. A continuación utilizaré unos pocos ejemplos que ilustran la forma como este colosal esfuerzo cambió la historia de la medicina.

1. Utilizar el modelo animal adecuado: se decidió utilizar perros beagle debido a que estos maravillosos animales eran utilizados por la Comisión de Energía Atómica de Estados Unidos para realizar experimentos, específicamente para observar los efectos de la exposición a radiación atómica. Por ende, existía la información más completa de los requerimientos nutricionales y de líquidos en esta raza de perros. Mantener a los animales vivos con líquidos y nutrientes podría ser considerado un éxito, pero lo realmente genial fue lograr el crecimiento y desarrollo normal de estos beagles desde las 8 semanas de vida a la adultez ${ }^{(1,2)}$. Este experimento cambió la percepción de lo que era posible y de manera contundente abrió la puerta para demostrar que esta nueva tecnología se podría utilizar para el crecimiento, desarrollo y no solo para el mantenimiento de la vida. Al final, su uso en neonatos lograría salvar la vida de millones de niños que no podían ser alimentados por vía enteral.

2. Acceso venoso: el acceso venoso usual en la práctica clínica requería el uso de agujas reusables y esterilizables; los catéteres plásticos disponibles comercialmente (polietileno) eran tóxicos para los tejidos lo que limitaba su uso por tiempo prolongado, lo mismo sucedía con los catéteres de teflón usados en el laboratorio. Además, la alta osmolaridad de estas soluciones debido a las concentraciones altas de solutos causaban un daño significativo en las venas periféricas. Esta combinación de factores que el doctor Dudrick vivió en carne propia cuando en el laboratorio intentó infundir la solución preparada por él a través de su propia vena y le causó una flebitis significativa, lo llevó a formular una respuesta que iba en contra de lo que creía el establecimiento médico, incluido su mentor, el doctor Rhoads, que el acceso de las venas centrales por tiempo prolongado era muy peligroso y estaba contraindicado ${ }^{(4)}$.

El Doctor Dudrick decidió entonces, utilizar la vena yugular $y$ de esta manera acceder a la vena cava superior. Finalmente, y como resultado de fallar y reintentar encontró que el policloruro de vinilo o PVC (obtenido en una ferretería y esterilizado) funcionaba mejor, y era más durable, que cualquier catéter comercial. Resolviendo de esta manera el problema de un acceso venoso central de largo plazo que permitiera la administración de soluciones concentradas, de uso rutinario en nuestros hospitales hoy en día.

3. Filtros: la dificultad para esterilizar estas soluciones hacían imposible la administración por vía intravenosa de los nutrientes prescritos; la caramelización producida al calentar la glucosa, en general los azúcares, a altas temperaturas impedía utilizar este método de esterilización. El doctor Dudrick encontró una solución muy ingeniosa: en la farmacia del Pennsylvania Hospital utilizaban filtros pequeños para eliminar la contaminación de soluciones oftálmicas de isótopos radioactivos (soluciones que no se pueden esterilizar en autoclave) estos filtros eran producidos por la compañía Millipore. El doctor Dudrick descubrió que ese tipo de filtros era utilizado también para evitar contaminaciones en la industria cervecera y vinícola, de modo que consiguió estos filtros industriales para esterilizar litros de su solución, los cuales permitían lograr soluciones estériles, pero cualquier contaminación en el aparato de infusión podía causar bacteriemia, así que se consiguieron filtros en "línea" o filtros en el sistema de infusión que serían utilizados antes de que el líquido entrara al individuo (Figura 1) ${ }^{(5)}$. Estos filtros fueron conseguidos en la industria de la gasolina ${ }^{(1)}$. El desarrollo de esta técnica y su implementación modificaron la medicina y ahora es difícil imaginar sistemas de infusión modernos sin filtros.

4. Bombas de infusión: el uso clínico de las bombas de infusión no era estándar, todas las infusiones en el hospital eran administradas por sistema de gravedad y medidas por el método de goteo. Esto llevó a que con frecuencia las enfermeras trataran de completar, al final del turno, el volumen ordenado aumentando la velocidad de infusión, lo que producía alteraciones metabólicas por las altas dosis de 
glucosa infundida. Esta situación motivó la utilización de bombas de infusión eléctricas rudimentarias (Figura 1). Implementar esta tecnología no estuvo exenta de dificultades, debido a que los cambios en la electricidad de corriente alterna, picos eléctricos, alteraban la velocidad de infusión de las bombas, y esta circunstancia llevó a diseñar, con la asistencia de la escuela de ingeniería, bombas de infusión con protección para picos de voltaje y alarmas de infusión. Estas bombas de infusión son estándar en los centros hospitalarios del mundo y hoy no podríamos imaginar la atención de nuestros pacientes sin ellas.

5. Camilla metabólica (Figura 1): alimentar el primer infante, una niña con diagnóstico de catástrofe intra-abdominal que no permitía ningún tipo de alimentación enteral fue un reto increíble que requirió el desarrollo de nuevas tecnologías no solo para el tratamiento sino para la monitoria de los pacientes, también generó problemas éticos nunca antes vistos. Sin embargo, me quiero enfocar en el desarrollo de una "camilla metabólica": los requerimientos precisos y la respuesta a nutrientes administrados en esta paciente debían ser calculados diariamente, para esto se diseñó una camilla que recogiera todas las excreciones de la paciente. Su medición permitía el ajuste en el tratamiento para proveer los nutrientes, líquidos y electrolitos óptimos. Este tipo de monitorización no solo incrementó el conocimiento de la fisiología metabólica en humanos, sino también, demostró la viabilidad y bondad de la terapia intensiva en medicina. Las vidas afectadas por tomar la decisión de implementar la nutrición parenteral total en una bebé cambiaron la manera de entender las posibilidades de la profesión médica.

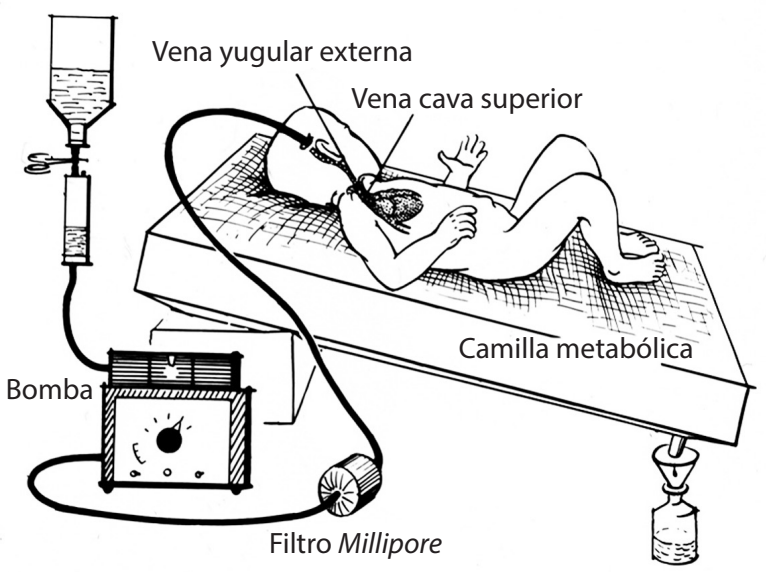

Figura 1. Camilla metabólica.

Muchas otras tecnologías o técnicas fueron implementadas y desarrolladas por este gigante de la cirugía y por su equipo; sin embargo, ningún espacio sería suficiente para nombrarlos a todos. Lo importante aquí
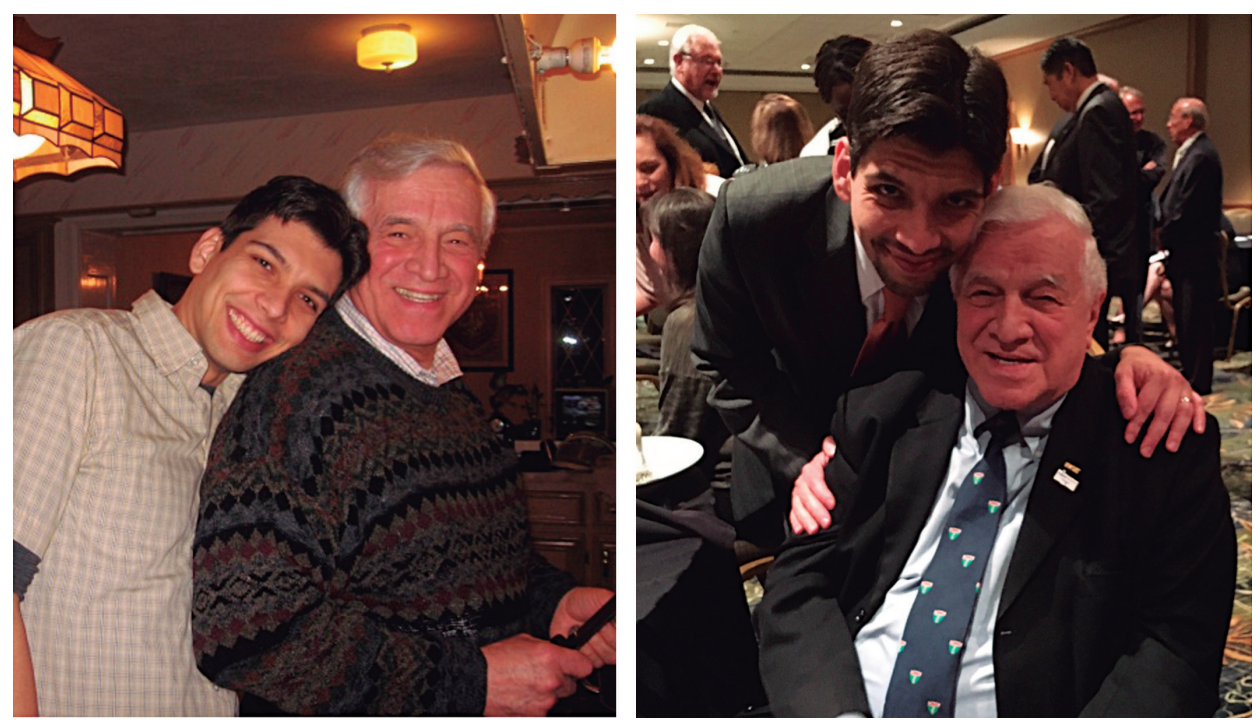

Figura 2. Doctores Stanley J. Dudrick y José Mario Pimiento. 
es reconocer que el trabajo del doctor Stanley John Dudrick, cambió la medicina para siempre. De la misma manera que pensamos que adelantos tecnológicos como la Internet, "Uber", cambiaron la manera de ver el mundo, me atrevo a decir que el descubrimiento de la Nutrición Parenteral Total y las tecnologías asociadas cambiaron el trayecto de la medicina moderna, exactamente lo que define Tecnología Disruptiva, como lo han sido innovaciones como la asepsia y antisepsia o como el descubrimiento de la anestesia general y los antibióticos.

Hoy recordamos y rendimos tributo a la vida de este titán (Figura 2), de este genial soñador que se adelantó a la época, cuyo maravilloso legado cambió el rumbo de la medicina, impactó y seguirá beneficiando a nuestros pacientes. ¡Vivirá por siempre Doctor Stanley John Dudrick!

\section{Referencias bibliográficas}

1. Gosche JR. Oral History Project: Stanley J. Dudrick. 2006. [Internet](consultado el 1 de febrero 2020) Disponible en: https://www.aap.org/en-us/about-the-aap/PediatricHistory-Center/Documents/Dudrick.pdf.

2. Dudrick SJ. Early developments and clinical applications of total parenteral nutrition. JPEN J Parenter Enteral Nutr. 2003;27(4):291-9.

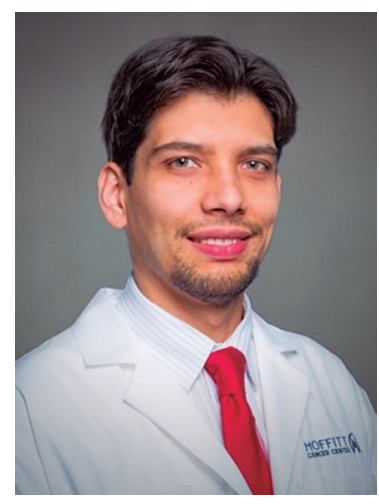

José Mario Pimiento, MD, FACS. Médico cirujano de la Universidad Nacional de Colombia. En el año 2000 conoció al doctor Dudrick durante la rotación como estudiante de medicina en el Hospital de Bridgeport, Connecticut. Entrenado en Cirugía General por el profesor Dudrick en el Stanley J Dudrick Department of Surgery en el hospital de Saint Mary's afiliado al Sistema de Yale New Heaven. Es cirujano oncológico con práctica enfocada en el tracto gastrointestinal alto en el Moffitt Cancer Center, Tampa, uno de los centros designados por el Instituto Nacional de Cáncer en USA. Ganador del premio Stanley Dudrick Research Scholar otorgado por la American Society for Parenteral and Enteral Nutrition (ASPEN) en el año 2019, estuvo en contacto permanente con su maestro y mentor.

3. Ab Rahman A, Abdul Hamid UZ, Chin T. Emerging Technologies with Disruptive Effects: A Review. PERINTIS eJournal. 2017;7:111-28.

4. Wilmore DF, Dudrick SJ. Safe long-term venous catheterization. Arch Surg. 1969;98(2):256-8.

5. Wilmore DW, Dudrick SJ. An in-line filter for intravenous solutions. Arch Surg. 1969;99(4):462-3. 